Open Access

Michael Pust*, Ekaterina Ivanova, Henning Schmidt and Jörg Krüger

\title{
Design of a pressure sensitive matrix for analyzing direct haptic patient-therapist interaction in motor rehabilitation after stroke
}

DOI 10.1515/cdbme-2017-0013

\begin{abstract}
Robot based therapy is one of the prevalent therapeutic approaches in motor stroke rehabilitation. It is often used in hospitals in combination with conventional therapy. In order to optimize human-robot interaction, we aim to investigate how a therapist physically supports patients during motor training of the upper extremities. This paper presents the design of a flexible textile sensor matrix, which measures the pressure exerted between therapist and patient during direct haptic interaction as well as the hand position and orientation in space. The matrix contains 144 sensors which enables measuring pressure intensity and localization of areas where the pressure is applied. The measurement matrix was evaluated with four healthy participants.
\end{abstract}

Keywords: haptics; human-human interaction; sensor system; stroke rehabilitation.

\section{Introduction}

Yearly approximately 262,000 first-time or repeated strokes occur in Germany [1] and 700,000 in the United States [2] respectively, making stroke one of the dominant causes of acquired disability. One of the clinically evaluated and effective therapies is robot based rehabilitation training [3-5]. Greater training time and a high intensity of repeated movements make rehabilitation robot based therapy very effective [3, 4]. However, a crucial factor for the therapeutic benefit of such devices is the implemented control

\footnotetext{
*Corresponding author: Michael Pust, TU Berlin, Berlin, Germany, E-mail: michael.pust@campus.tu-berlin.de

Ekaterina Ivanova: Rehabilitation Robotics Group, Chair of Industrial Automation Technology, TU Berlin, Berlin, Germany, E-mail: ivanova@ifw.tu-berlin.de

Henning Schmidt: Rehabilitation Robotics Group, Fraunhofer Institute for Production Systems and Design Technology, Berlin, Germany

Jörg Krüger: Chair of Industrial Automation Technology, TU Berlin, Berlin, Germany
}

algorithms which define the haptic interaction between the patient and the robot [6]. A large variety of control algorithms has been developed recently [5-7] and it is known that different control strategies influence motor recovery in different ways $[6,8]$. However, it is not yet clear which algorithm is the best option for motor rehabilitation $[6,7]$ and how new algorithms should be designed to ensure optimal recovery.

So far the way therapists perform haptic "assist as needed" interaction with a patient during physiotherapy is seen as the gold standard. Hence, our hypothesis is that a control strategy, which supports a patient in the way a therapist would do, would be most beneficial for motor learning. Some studies have already shown that humanhuman interaction is more efficient for executing motor tasks than working alone $[9,10]$ and even more efficient than working with a robotic partner, regarding the time needed to accomplish a task [11,12]. Moreover, achieving a task in haptic connection with another person facilitates motor learning for healthy subjects more than achieving such a task either without any interaction partner [10] or with a robotic partner [11]. Furthermore, understanding the therapist's haptic behaviour can improve the mechanic design of future robotic therapeutic devices.

Therefore, we assume that closely investigating humanhuman interaction during performance of rehabilitation training and subsequent transfer of the haptic behaviour to a rehabilitation robot controller improves interaction between the patient and a robotic device for a similar task. To study direct haptic patient-therapist interaction, we need a sensor system that can measure pressure on the hand surface as well as hand position and orientation. At the same time, the sensor system should not inhibit the therapist-patient interaction. The system should be easy and flexible to apply on either right or left hand (depending on the patient's affected side). Our goal was to analyse supination/pronation and flexion/extension movements, so the future sensor system needs to cover the hand (excluding fingers), wrist and parts of the forearm. The sensor system should be able to localize the interaction points and should be big enough to measure pressure 
distribution on inner and outer part of the hand. Finding a low cost solution was also a relevant aspect.

We found one existing solution to investigate comparable research questions for lower extremities: Galvez et al. [13] developed a lower leg orthosis which comprises two six-axis force/torque sensor and accelerometer units equipped with handles. The measurement units are attached close to knee and ankle and measure the therapeutic motion and interaction force support during gait training. However, the system does not allow measurement of force distribution on hand or leg surface. Other possible solutions is the pliance ${ }^{\circledR}$ sensing glove by Novel GmbH or the medilogic ${ }^{\circledR}$ sensor mat by T\&T medilogic Medizintechnik GmbH. The systems allow analysing the interaction pressures with the help of capacitive transducers. However, measurements of the hand position are not possible. Existing solutions, which are also rather expensive, do not meet all of the mentioned requirements. Therefore, it was decided to develop a specific sensor system. In this paper, we present a new soft pressure sensitive matrix for measurement of interaction pressure with integrated inertial sensors for estimating the hand position.

\section{Technical specification}

This article focuses on the direct measurement of pressures in human-human interaction by using a high density of thin layer pressure sensors. Figure 1 shows an overview of such a measurement matrix with electronic components. Inertial sensors are used to track position and orientation of the patients' hand. To measure the haptic interaction between patient and therapist during a rehabilitation session, the matrix is attached with three velcro fasteners around the arm and hand of the patient. The sensor system's slim structure does not restrict movements in any way. This

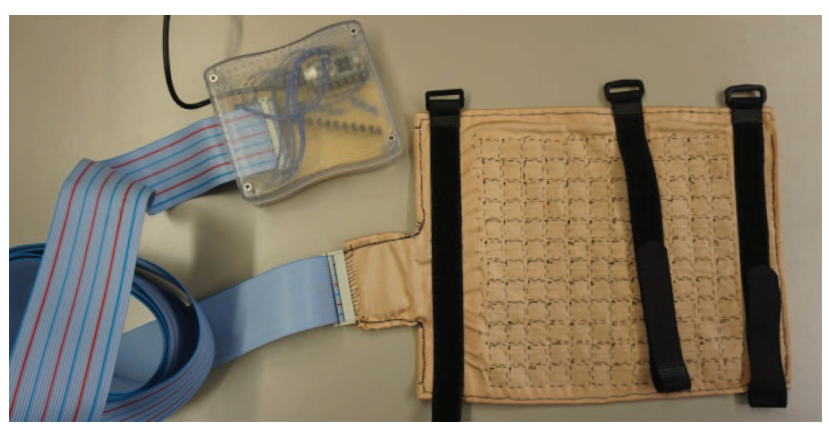

Figure 1: Overview of the measurement matrix. permits the therapist to support the patient during the experiment as if it was an ordinary training session.

The sensitive matrix has a size of $20 \times 20 \mathrm{~cm}$. It contains 144 pressure sensors and two position sensors. Pressure sensor elements are arranged in a $12 \times 12$ Matrix which is connected through a multiplex circuit. The size of each pressure sensors matrix array is approx. $1 \mathrm{~cm}^{2}$. A conductive film by 3M (also known as Velostat ${ }^{\circledR}$ or Linqstat ${ }^{\circledR}$ ) is used as pressure sensitive material. In combination with single layers of conductive thread, conductive fabric and non-conductive fabric, the different layers build one sensor element (as shown in Figure 2). Two LSM9DS0 sensors by STMicroelectronics are used to compute the position. It is a SIP (system-in-package) with integrated linear acceleration sensor, an angular rate sensor and a magnetic sensor. Each sensor collects data for the orientation in three dimensions to determine the angles for the movements. One sensor is attached on the upper site of the matrix to capture the hand position and a second sensor is attached on the lower site of the arm, so that the position of hand, forearm and the angle between them are captured.

The circuit is controlled by the evaluation board “Teensy 3.2" by PJRC and is programmed with Arduino software. This is a very small evaluation board with a high number of analogue and digital GPIO's (general purpose input output) and is used to measure the analogue signal of the force sensors and the digital signals from the inertial sensors as well as pre-processing the signals. A major advantage of this sensor system is its low material costs (approximately $150 €$ ), compared to the price of similar systems.

The captured data is transferred via USB to a computer running MATLAB ${ }^{\circledR}$ software which provides the calibration data needed to calculate the forces and the position. The force sensitive matrix is calibrated with weights ranging from 10 to 1000 gram $(0.0981 \mathrm{~N}$ to $9.81 \mathrm{~N})$. For data processing and visualization, a very simple graphical hand model was implemented in MATLAB ${ }^{\circledR}$. The fundamental form of the model is a cylinder, which is calculated by three measured dimensions from the subject: 1) circumference

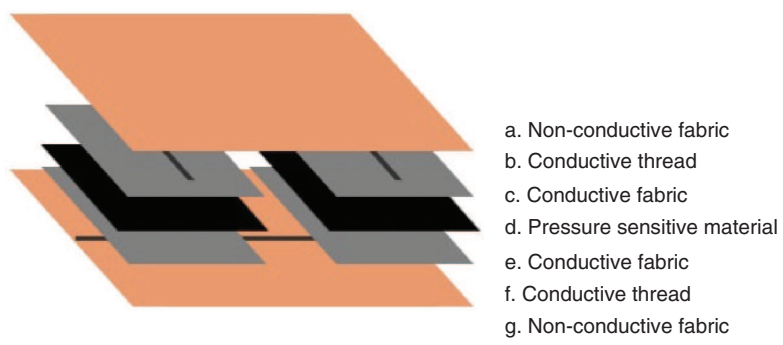

Figure 2: Layers of one sensor element. 


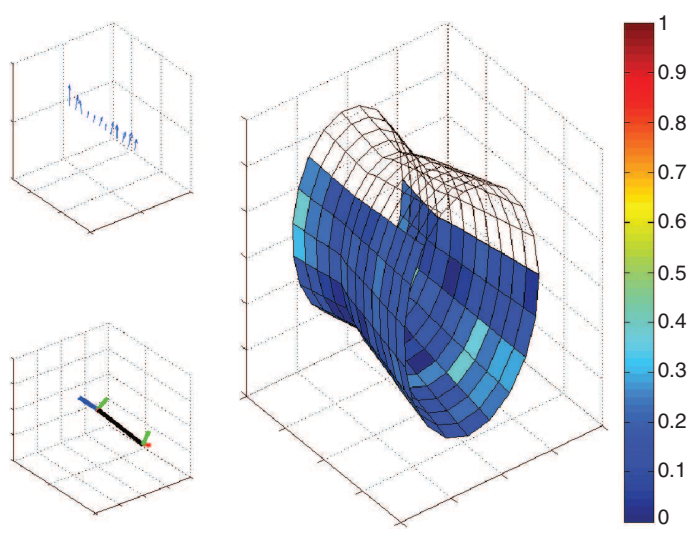

Figure 3: Visualization of processed data: top left - vector forces; down left - position of the hand; right - normalized values of sensors indicated with color.

around hand, 2) circumference around wrist and 3) circumference around arm. Depending on the subject's arm and hand dimensions, there could be a gap between the first and the last sensor lines as matrix size is limited. As shown on the right side of the visualization in Figure 3, the 144 sensor elements are placed around the cylinder while the gap is filled with empty placeholders calculated by the software. A relative colour scale represents impacts of forces on single elements, ranging from dark blue for low pressure, to dark red for high pressure. The top left plot in the visualization shows the direction of merged forces around hand and arm. The bottom left plot shows the orientation of arm and hand in relation to a fixed position in the plot which is given by the sensor on the arm. Haptic data evaluated by this matrix is saved in a csv data file for subsequent analysis.

\section{Evaluation}

To evaluate the functionality of the sensor system, we tested the matrix with healthy subjects. Four healthy subjects participated in the evaluation: two males, two females, all right handed, and in the age range of 24 to 29 years (mean age is 27 years). The study focused on the portability and quality of the measurements by comparing different hand and arm sizes. The two female subjects had similar hand and arm sizes. The hand and arm sizes of the two males were also similar to each other.

Each subject was asked to perform two movements: pronation and supination of the forearm between $-90^{\circ}$ to $90^{\circ}$ around a fixed axis, and tension and extension of the hand between $-60^{\circ}$ to $60^{\circ}$ around the wrist. A scale with marks at $0^{\circ}, 30^{\circ}, 60^{\circ}, 90^{\circ}$ on the horizontal and vertical area was used as a reference for the measurements (shown in Figure 4).

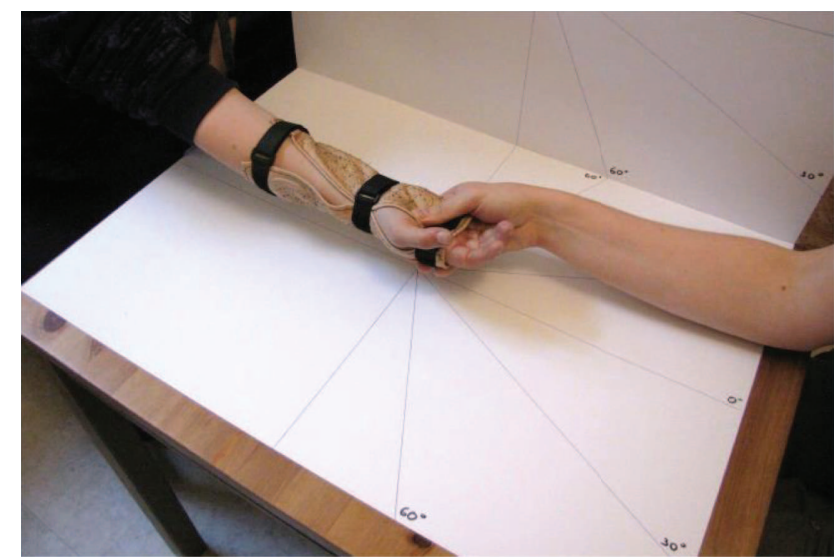

Figure 4: Evaluation setup, lines define angel of rotation movement in horizontal and vertical plane.

To define an angle position for tension and extension data of both inertial sensors on arm and hand were used. The arm was kept in a horizontal position while the hand was moved. The determined angles are relative values for the orientation of the hand to the arm and are close to the expected range. Data of the arm sensor was used for pronation and supination. The angles were measured as relative values for the rotation around the middle axes of the arm in relation to a fixed starting position. These values are prone to differ significantly because the hand was aligned by eye in this setup.

The movements were performed with the left and the right arm in two phases: At first individuals executed the task actively without being helped by someone else. During the next phase, the passive phase, the same movements were completed by the trial manager while the subject's limb was not tensed.

The minimum and maximum values for the angles along with the values for the maximum pressures on one square centimetre (dimension of one sensor element) in passive implementation of the movement are shown in Table 1 for all four subjects. During the active phase, only small pressures were measured due to the movement of the matrix itself. These pressures is not applied from outside as they are results of the participants own movement.

The data did not reveal any direct dependence concerning the hand sizes and left or right arm. However, statements about dependencies based on only four subjects are not convincing and evaluation with more participants is required. Table 1 gives an overview of the maximum interaction pressure between two interacting persons: trial manager and respective subject. The table shows that the interaction pressure is depending on the movement type and differs between subjects. 
Table 1: Participants data, angle values and maximum pressures of passive movements.

\begin{tabular}{lrrrr}
\hline Participants & P01 & P02 & P03 & P04 \\
\hline $\begin{array}{l}\text { Gender } \\
\text { Hand/Forearm } \\
\text { circumference, } \\
{[\mathrm{cm}]}\end{array}$ & $21 / 25$ & $22 / 26$ & $28 / 25$ & $21 / 25$ \\
$\begin{array}{l}\text { Pressure P/S, } \\
{\left[\mathrm{N} / \mathrm{cm}^{2}\right]}\end{array}$ & 3.35 & 8.77 & 5.39 & 5.76 \\
$\begin{array}{l}\text { Pressure T/E, } \\
{\left[\mathrm{N} / \mathrm{cm}^{2}\right]}\end{array}$ & 2.15 & 2.98 & 2.67 & 4.44 \\
$\begin{array}{l}\text { Angles P/S, } \\
{\left[{ }^{\circ}\right][\mathrm{min} / \mathrm{max}]} \\
\text { Angles T/E, }\end{array}$ & $-62.6 / 41.1$ & $-60.1 / 62.0$ & $-49.5 / 49.7$ & $-56.1 / 61.5$ \\
{$\left[{ }^{\circ}\right][\mathrm{min} / \mathrm{max}]$} & $-102 / 18$ & $-47.1 / 89.4$ & $-71.1 / 53.3$ & $-42.1 / 81.2$ \\
\hline
\end{tabular}

$\mathrm{P} / \mathrm{S}$, pronation/supination; $\mathrm{T} / \mathrm{E}$, tension/extension.

\section{Discussion and future work}

While capturing data of the first two subjects, three circumferences were measured around the attached matrix. These circumferences are needed for the hand model. This leads to a small deviation from the calculated model and from real values. It was also observed that the dimensions of the sensor matrix are too small to fit around large hands. This makes it impossible to measure large hands as the gap in the model would be too big to allow a sufficient calculation of merged pressure. To solve this problem, a larger matrix with more sensor elements is needed.

Furthermore, we detected interfering signals due to small pressures, produced by the folded soft sensor matrix. This problem could be solved by signal processing. Besides that, using velcro fasteners to attach the matrix and the position of the cable itself were criticized as unpleasant by evaluation participants. The attachment of the sensor system should therefore be revised. For example, a different manufacturing method and another sensor material could provide more flexibility.

The position and orientation capturing is optimized for collecting data of described movements and is calculated only with accelerometer data of the inertial sensors in relation to fixed points and a fixed coordinate system. Improving the algorithm, so that it additionally uses data of all integrated LSM9DS0 sensors, will allow a more complex evaluation of the position data and therefore also enable a better movement analysis. This improved algorithm would also allow to collect data during complex motion sequences with position tracking in 3D space.

The calibration process of the system is quite complex because each sensor should be calibrated separately and is thus very time consuming. Calibration could be improved with devices like trublu ${ }^{\circledR}$ from novel GmbH.

In this paper, we presented the design and evaluation of a pressure sensitive matrix for data capturing of interaction forces between therapist and patient, and positions of the hand during a rehabilitation session. In our future work, we plan to modify the sensor system for ergonomic application on the subject's hand. The next step will be capturing and analysing the haptic data of patient-therapist interaction in a clinical environment.

Acknowledgment: The authors wish to thank Ksenia Miroshnichenko, Georg Schott and Christian Wiertelorz for support during design of the first sensor system prototype and research therapists of rehabilitation clinic Medical Park Berlin for clinical support.

\section{Author's Statement}

Research funding: This research is part of the project BeMobil founded by Federal Ministry of Education and Research of Germany. Conflict of interest: Authors state no conflict of interest. Informed consent: Informed consent has been obtained from all individuals included in this study. Ethical approval: The research related to human use complies with all the relevant national regulations, institutional policies and was performed in accordance with the tenets of the Helsinki Declaration.

\section{References}

[1] Heuschmann PU, Busse O, Wagner M, Endres M, Villringer $M$, Röther J, et al. Schlaganfallhäufigkeit und Versorgung von Schlaganfallpatienten in Deutschland. Akt Neurol. 2010;37:333-40.

[2] Ingall T. Stroke - incidence, mortality, morbidity and risk. J Insur Med. 2003;36:143-52.

[3] Kwakkel G, Kollen B, Krebs H. Effects of Robot-assisted therapy on upper limb recovery after stroke: a systematic review. Neurorehabil Neural Repair. 2008;22:111-21.

[4] Norouzi-Gheidari N, Archambault P, Fung J. Effects of robotassisted therapy on stroke rehabilitation in upper limbs: systematic review and meta-analysis of the literature. J Rehabil Res Dev. 2012;49:479-96.

[5] Prange G, Jannink M, Groothuis-Oudshoorn C, Hermens H, ljzerman M. Systematic review of the effect of robot-aided therapy on recovery of the hemiparetic arm after stroke. J Rehabil Res Dev. 2006;43:171-84.

[6] Marchal-Crespo L, McHughen S, Cramer S, Reinkensmeyer D. The effect of haptic guidance, aging, and initial skill level on motor learning of a steering task. Exp Brain Res Springer. 2010;201:209-20. 
[7] Basteris A, Nijenhuis S, Stienen A, Buurke J, Prange G, Amirabdollahian F. Training modalities in robot-mediated upper limb rehabilitation in stroke: a framework for classification based on a systematic review. J Neuroeng Rehabil. 2014;11:1.

[8] Cai L, Fong A, Otoshi C, Liang Y, Burdick J, Roy R, et al. Implications of assist-as-needed robotic step training after a complete spinal cord injury on intrinsic strategies of motor learning. J Neurosci. 2006;26:10564-8.

[9] Reed K, Peshkin M, Hartmann M, Grabowecky M, Patton J, Vishton P. Haptically linked dyads are two motor-control systems better than one? Psychol Sci. 2006;17:365-6.
[10] Wegner N, Zeaman D. Team and individual performances on a motor learning task. J Gen Psychol. 1956;55:127-42.

[11] Ganesh G, Takagi A, Osu R, Yoshioka T, Kawato M, Burdet E. Two is better than one: Physical interactions improve motor performance in humans. Sci Rep. 2014;4:3824.

[12] Reed K, Peshkin M. Physical collaboration of human-human and human-robot teams. IEEE Trans Haptics. 2008;1:108-20.

[13] Galvez J, Kerdanyan G, Maneekobkunwong S, Weber R, Scott $M$, Harkema S, et al. Measuring human trainers' skill for the design of better robot control algorithms for gait training after spinal cord injury. Proc. 9th International Conference on Rehabilitation Robotics, 2005. ICORR; 2005. p. 231-4. 